\title{
Advantages and Disadvantages of Hyperbaric Oxygen Treatment in Mice with Obesity Hyperlipidemia and Steatohepatitis
}

Koichi Tsuneyama, ${ }^{1}$ Yen-Chen Chen, ${ }^{2}$ Makoto Fujimoto, ${ }^{3}$ Yoshiyuki Sasaki, ${ }^{4}$ Wataru Suzuki, ${ }^{5}$ Tsutomu Shimada, ${ }^{5}$ Seiichi lizuka, ${ }^{5}$ Mitsunobu Nagata, ${ }^{5}$ Masaki Aburada, ${ }^{5}$ and Shao-Yuan Chen ${ }^{6,7}$

${ }^{1}$ Department of Diagnostic Pathology, Graduate School of Medicine and Pharmaceutical Sciences, University of Toyama, Toyama, Japan

${ }^{2}$ Department of Microbiology and Immunology, National Defense Medical Center, Taipei, Taiwan

${ }^{3}$ Department of Japanese Oriental Medicine, Graduate School of Medicine and Pharmaceutical Sciences, University of Toyama, Toyama, Japan

${ }^{4}$ Institute for Animal Reproduction, 1103 Fukaya, Kasumigaura-shi, Ibaraki 300-0134, Japan

${ }^{5}$ Faculty of Pharmacy, Research Institute of Pharmaceutical Sciences, Musashino University, 1-1-20 Shinmachi, Nishitokyo-shi, Tokyo 202-8585, Japan

${ }^{6}$ School of Medicine, Fu Jen Catholic University, New Taipei City, Taiwan

${ }^{7}$ Department of Hyperbaric Medicine and Neurology, Cardinal Tien Hospital, New Taipei City, Taiwan

Received 30 August 2011; Accepted 15 October 2011

Academic Editors: M. Elisaf and E. Macken

The effect of hyperbaric oxygen treatment (HBOT) was examined using MSG mice, which are an animal model of obesity, hyperlipidemia, diabetes, and nonalcoholic fatty liver disease. Nineteen MSG male mice were divided into HBOT treated and control groups at 12 weeks of ages. The HBOT group was treated with hyperbaric oxygen from 12 to 14 weeks (first phase) and then from 16 to 18 weeks (second phase). Interestingly, the body weight of the HBOT group was significantly lower $(P<0.01)$ than that of the control group. In contrast, the serum lipid level did not show significant changes between the two groups. As for the effects of increasing oxidative stress, the liver histology of the HBOT group showed severer cellular damage and aberrant TNF- $\alpha$ expression. HBOT has the advantage of improving obesity in patients with metabolic syndrome, but the fault of causing organ damage by increasing oxidative stress.

KEYWORDS: Nonalcoholic steatohepatitis, obesity, hyperbaric oxygen treatment, MSG mice

Correspondence should be addressed to Shao-Yuan Chen, sychen@ndmctsgh.edu.tw Copyright (c) 2011 Koichi Tsuneyama et al. This is an open access article distributed under the Creative Commons Attribution License, which permits unrestricted use, distribution, and reproduction in any medium, provided the original work is properly cited.

Published by TheScientificWorldJOURNAL; http://www.tswj.com/ 


\section{INTRODUCTION}

"Metabolic syndrome" is serious current issue. Metabolic syndrome is called "visceral fat syndrome" because visceral fat accumulation and macrophage aggregations described as "crown-like structures" occur primarily and then cause various intractable disorders to the whole body [1,2]. The liver phenotype of metabolic syndrome is called "nonalcoholic fatty liver disease (NAFLD)" and nonalcoholic steatohepatitis (NASH), which is the critical type of NAFLD, is a refractory progressive disease, which progresses to liver cirrhosis or hepatocellular carcinoma [3-5]. Although the elucidation of the mechanism behind the pathological condition remains controversial, the "two-hit theory" has support as the pathogenetic mechanism of NASH. It is suggested that simple steatosis arises as a result of the first hit and that the pathologic condition progresses due to a second hit such as oxidative stress [6]. This means that oxidative stress may be an exacerbating factor of liver disease caused by metabolic syndrome.

Hyperbaric oxygen therapy (HBOT) is a remedy in which an atmospheric pressure environment higher than the normal atmosphere is created artificially, and the patient inhales hyperbaric oxygen continuously in the environment in order to increase the level of dissolved oxygen in their blood, which promptly acts to improve systemic or local hypoxia [7]. This remedy is usually employed for conditions such as carbon monoxide poisoning, limb arterial occlusive diseases, diseases of the central nervous system, sudden deafness, and retinal arterial occlusion. Recently, its application has been expanded to multiple fields including orthopedics $[8,9]$ and sports medicine $[10,11]$. We have proposed the possibility that HBOT would be effective in the treatment of autoimmune diseases and hematopoietic malignancies $[12,13]$. These effects are supposedly caused by the strong oxidative stress that is produced as a side effect of HBOT. We hypothesized that oxidative stress predominantly affects autoreactive lymphoid and tumor cells. Various inflammatory cytokines play an important role in the onset and progression of metabolic syndrome and NASH [14]. The visceral fat cells of obese patients store several times more fat than those of nonobese patients. These cells are often enlarged, and their cell membranes frequently collapse causing the leakage of stored lipids. Subsequently, macrophages begin to cluster in order to process these lipids. These clustered macrophages are called "crown-like structures" and are frequently observed in the visceral fat of patients with metabolic syndrome $[15,16]$. It is thought that the various cytokines and chemokines that are produced by these macrophages in visceral fat are closely related to the onset and exacerbation of the symptoms of metabolic syndrome. They are also considered to be involved in the formation of hepatic lesions through the portal vein. It is expected that when HBOT is used for the treatment of metabolic syndrome [17-19], these excessively activated cytokines may be inhibited, at the same time, as metabolism is accelerated by the more efficient oxygen supply. On the other hand, the risk that NASH will be worsened by increased oxidative stress is also taken into consideration [20-22]. In this study, using MSG mice with NASH and a background of obesity, hyperlipidemia, and type-2 diabetes which are all symptoms of metabolic syndrome, the effects of HBOT on obesity, hyperlipidemia, and NASH are examined [23, 24].

\section{MATERIALS AND METHODS}

\subsection{Experimental Animals}

The MSG mouse is an obese animal model, which is prepared by hypodermic injection of monosodium glutamate (MSG) into an ICR mouse immediately after birth. This mouse is reported to show symptoms of obesity, type-2 diabetes, hyperlipidemia, and non-alcoholic fatty liver disease after usual feeding without showing hyperphagia [23, 24]. Although it is known that MSG mice of both sexes demonstrate various symptoms of metabolic syndrome, males show more severe symptoms. MSG mice are usually prepared by administering a hypodermic injection of MSG of $2 \mathrm{mg}$ per $\mathrm{g}$ of body weight for successive five days. Recently, Sasaki et al. reported that MSG mice that were prepared by one hypodermic injection of $4 \mathrm{mg}$ MSG showed a more severe pathologic condition than those produced using the conventional method [25]. In present study, 19 MSG male mice were prepared according to the method of Sasaki et al. 


\subsection{Study Design}

Nineteen MSG male mice were divided into the HBOT-treated group $(n=10)$ and the control group (HBOT-untreated group) $(n=9)$ at 12 weeks of age. The HBOT-treated group was treated with HBOT ( $2.5 \mathrm{ATA} / 60 \mathrm{~min} / \mathrm{d}$ ) over 2 weeks from 12 to 14 weeks of age for 1 hour a day (first phase). Then, after a two-week interval, the mice were treated with HBOT (2.5 ATA/60 min/d) for another two weeks from 16 to 18 weeks of ages for 1 hour a day (second phase). The control group was kept in a chamber under normal pressure instead of HBOT for 1 hour and supplied with a normal concentration of oxygen. The mice in each group were sacrificed after 12 hours of fasting after the last HBOT treatment, and their serum, liver, and visceral fat were extracted.

\subsection{Measured Parameters}

Weekly weight was measured from the start of the experiment until sacrifice. In serum, triglycerides (TG), total cholesterol (T-chol), very-low-density lipoprotein (VLDL-) chol, low-density lipoprotein (LDL-) chol, and high-density lipoprotein cholesterol (HDL-) chol were measured. The cholesterol and phospholipid profiles in serum lipoproteins were analyzed using a dual-detection HPLC system with two tandemconnected TSKgel LipopropakXL columns (300 mm × $7.8 \mathrm{~mm}$; Tosoh, Tokyo, Japan) at Skylight Biotech (Akita, Japan).

Formalin-fixed paraffin-embedded specimens of the visceral fat and liver were prepared and analyzed pathomorphologically. Histopathological findings of the liver were scored using the NASH Clinical Research Network Scoring System based on four semiquantitative factors: steatosis [0-3], lobular inflammation [0-3], hepatocyte ballooning [0-2], and fibrosis [0-4] as previously described [26]. The NAFLD activity score (NAS) was defined as the unweighted sum of the scores for steatosis, lobular inflammation, and hepatocyte ballooning; thus, the scores ranged from 0 to 8. A NAS of 0 to 2 was considered not diagnostic of steatohepatitis, and scores of 5 or greater were taken as diagnostic of steatohepatitis.

\subsection{Immunohistochemistry}

Five $\mu \mathrm{m}$-thick specimens from each sample were immunostained using standard immunostaining procedures. Rat monoclonal antibody against mouse MAC-2 (Galectin-3) (Cedarlane, Hornby, Ontario; $1: 100$ dilutions) was used as a marker of macrophages. Rabbit polyclonal antibody against mouse TNF$\alpha$ (Monosan, Uden, The Netherlands; $1: 20$ dilutions) was also used. Histofine-peroxidase for rat primary antibody (Nichirei, Tokyo, Japan) and Envision-peroxidase (Envision-PO) (DAKO, Glostrup, Denmark) for rabbit primary antibodies were used as secondary antibodies. For the substrate peroxidase, 3,3'diaminobenzidine (DAB) was applied. In all cases, optimal dilutions were used, and positive and negative samples were included in each assay.

\subsection{Statistical Analysis}

Statistical analysis was performed with Stat View version 5.0 (Abacus Concept, Berkley, Calif, USA). Data were expressed as mean \pm SE. The differences between the control and HBOT groups were analyzed using the Student's $t$-test. A value of $P<0.05$ was considered statistically significant.

\section{RESULTS}

\subsection{Change of Weight}

The body weights of the male MSG mice prepared using a single subcutaneous injection of MSG at a dose of $4 \mathrm{mg}$ per g body weight increased constantly until 20 weeks of age and then plateaued until 28 weeks 


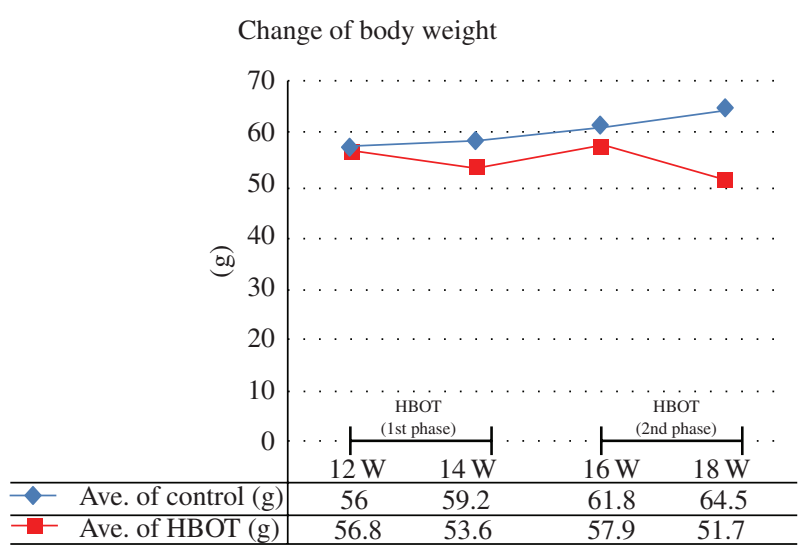

FIGURE 1: Change in body weight.

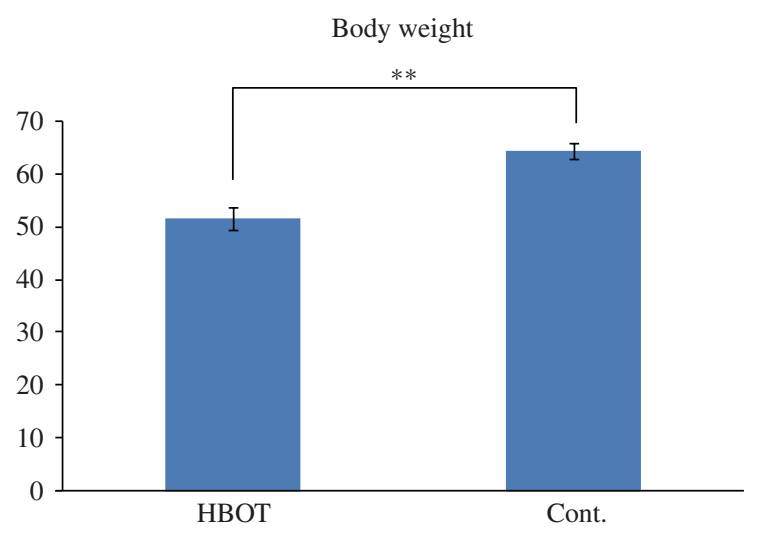

FIGURE 2: The weight of the HBOT group was significantly decreased compared with that in the control group (Student's $t$-test, ${ }^{* *} P<0.01$ ).

of age. Between 12 and 18 weeks of age, the rate of weight gain hits its peak with a mean of about a $9 \mathrm{~g}$ increase in weight. The changes in body weight in this study are shown in Figure 1. In the control group, the mean weight was $56.0 \mathrm{~g}$ at 12 weeks old, $59.2 \mathrm{~g}$ at 14 weeks old, $61.8 \mathrm{~g}$ at 16 weeks old, and $64.5 \mathrm{~g}$ at 18 weeks old, showing a constant increase. On the other hand, in the HBOT-treated group, the mean weight was $56.8 \mathrm{~g}$ at 12 weeks old but decreased rapidly to $53.6 \mathrm{~g}$ at 14 weeks old, that is, immediately after completing the first phase of HBOT. The mean weight increased again in the period of HBOT pause, and the mean weight was $57.9 \mathrm{~g}$ at 16 weeks old; however, it was $51.7 \mathrm{~g}$ at 18 weeks old, which is after the second phase of HBOT. The weight of the HBOT group was significantly decreased compared with that in the control group (Figure 2).

\subsection{Serum Lipid}

The serum lipid levels of each group are shown in Table 1 and Figure 3. In the control group, TG was $49.3 \mathrm{mg} / \mathrm{dL}$, T-chol was $192.9 \mathrm{mg} / \mathrm{dL}$, VLDL-chol was $8.7 \mathrm{mg} / \mathrm{dL}$, LDL-chol was $41.5 \mathrm{mg} / \mathrm{dL}$, and HDLChol was $142.7 \mathrm{mg} / \mathrm{dL}$. On the other hand, in the HBOT-treated group, TG increased to $61.6 \mathrm{mg} / \mathrm{dL}$. Although T-Chol declined slightly to $175.1 \mathrm{mg} / \mathrm{dL}$, VLDL-chol was $7.4 \mathrm{mg} / \mathrm{dL}$, LDL-chol was $45.1 \mathrm{mg} / \mathrm{dL}$, and HDL-chol was $121.7 \mathrm{mg} / \mathrm{dL}$. Although there were no statistical differences between the groups, LDLchol was higher and HDL-chol was lower in the HBOT-treated group. 
TABLE 1: The serum lipid.

\begin{tabular}{|c|c|c|c|c|c|c|c|c|c|c|}
\hline \multirow[b]{2}{*}{ Sample } & \multicolumn{5}{|c|}{ Cholesterol (mg/dL) } & \multicolumn{5}{|c|}{$\mathrm{TG}(\mathrm{mg} / \mathrm{dL})$} \\
\hline & Total & $\begin{array}{c}\mathrm{CM} \\
(>80 \mathrm{~nm})\end{array}$ & $\begin{array}{c}\text { VLDL } \\
(30-80 \mathrm{~nm})\end{array}$ & $\begin{array}{c}\text { LDL } \\
(16-30 \mathrm{~nm})\end{array}$ & $\begin{array}{c}\text { HDL } \\
(8-16 \mathrm{~nm})\end{array}$ & Total & $\begin{array}{c}\mathrm{CM} \\
(>80 \mathrm{~nm})\end{array}$ & $\begin{array}{c}\text { VLDL } \\
(70-80 \mathrm{~nm})\end{array}$ & $\begin{array}{c}\text { LDL } \\
(16-30 \mathrm{~nm})\end{array}$ & $\begin{array}{c}\text { HDL } \\
(8-16 \mathrm{~nm})\end{array}$ \\
\hline HBOT-1 & 217.70 & 3.06 & 15.48 & 52.19 & 146.98 & 165.50 & 27.59 & 84.87 & 19.04 & 34.00 \\
\hline НВOT-2 & 155.22 & 0.53 & 4.36 & 26.41 & 123.92 & 36.12 & 2.95 & 17.80 & 10.21 & 5.16 \\
\hline HBOT-3 & 111.52 & 1.05 & 6.32 & 15.74 & 88.41 & 51.20 & 6.58 & 27.34 & 9.82 & 7.47 \\
\hline HBOT-4 & 107.11 & 1.13 & 9.94 & 13.90 & 82.14 & 90.72 & 9.38 & 59.87 & 13.56 & 7.90 \\
\hline HBOT-5 & 166.60 & 0.88 & 11.72 & 37.44 & 116.56 & 78.42 & 5.79 & 49.83 & 15.12 & 7.67 \\
\hline HBOT-6 & 205.66 & 0.39 & 3.28 & 66.57 & 135.42 & 24.46 & 1.44 & 9.68 & 7.69 & 5.64 \\
\hline HBOT-7 & 207.75 & 0.25 & 3.72 & 59.95 & 143.82 & 22.55 & 0.86 & 9.86 & 7.63 & 4.20 \\
\hline HBOT-8 & 193.78 & 0.43 & 3.82 & 57.63 & 131.90 & 21.62 & 0.74 & 9.65 & 6.40 & 4.84 \\
\hline HBOT-9 & 245.03 & 1.73 & 9.21 & 98.57 & 135.53 & 71.69 & 12.76 & 36.07 & 12.66 & 10.20 \\
\hline HBOT-10 & 140.91 & 0.40 & 5.65 & 22.38 & 112.48 & 53.57 & 3.08 & 30.37 & 14.58 & 5.54 \\
\hline Ave & 175.13 & 0.99 & 7.35 & 45.08 & 121.72 & 61.59 & 7.12 & 33.53 & 11.67 & 9.26 \\
\hline Cont-1 & 166.30 & 0.19 & 15.56 & 34.86 & 115.69 & 63.74 & 1.82 & 49.69 & 8.31 & 3.92 \\
\hline Cont-2 & 128.11 & 0.03 & 3.61 & 24.80 & 99.67 & 28.63 & 0.36 & 18.14 & 6.81 & 3.32 \\
\hline Cont-3 & 324.08 & 0.03 & 10.41 & 94.72 & 218.93 & 86.88 & 0.58 & 60.97 & 16.51 & 8.82 \\
\hline Cont-4 & 189.40 & 0.04 & 7.25 & 32.47 & 149.63 & 52.36 & 0.39 & 34.64 & 11.99 & 5.34 \\
\hline Cont-5 & 133.15 & 0.05 & 6.09 & 20.81 & 106.20 & 35.94 & 0.46 & 26.42 & 5.94 & 3.13 \\
\hline Cont- 6 & 165.41 & 0.14 & 10.74 & 33.28 & 121.25 & 40.28 & 0.80 & 28.52 & 8.25 & 2.72 \\
\hline Cont-7 & 193.70 & 0.03 & 3.93 & 50.60 & 139.13 & 33.99 & 0.00 & 14.84 & 8.96 & 10.19 \\
\hline Cont-8 & 183.32 & 0.02 & 7.60 & 34.33 & 141.38 & 37.74 & 0.03 & 25.99 & 7.88 & 3.84 \\
\hline Cont-9 & 252.71 & 0.11 & 13.02 & 47.22 & 192.36 & 64.42 & 0.93 & 49.29 & 10.44 & 3.76 \\
\hline Ave & 192.91 & 0.07 & 8.69 & 41.45 & 142.69 & 49.33 & 0.60 & 34.28 & 9.45 & 5.00 \\
\hline
\end{tabular}

\subsection{Histopathological Imaging of Visceral Fat}

In the control group, visceral fat was markedly enlarged, and crown-like structures were also observed in some places (Figure 4(a)). These represented MAC-2-positive macrophage aggregation (Figure 4(b)). On the other hand, in the HBOT-treated group, the lipid droplets were smaller, and the number of crown-like structures tended to be lower (Figures 4(c) and 4(d)).

\subsection{Histopathological Imaging of the Liver}

In the control group, fatty changes were recognized in the hepatocytes in the Zone 3 to Zone 2 regions. Ballooning degeneration was also found in various locations (Figure 5(a)). TNF- $\alpha$ expression was not seen in hepatocytes or Kupffer cells (Figure 5(b)). Although the emergence of lipogranuloma and focal necrosis was also observed and the image was considered to be equivalent to human NASH, no Mallory bodies or megamitochondria were evident and no fibrosis was seen either. On the other hand, in the HBOT-treated group, although the level of fatty change was slightly decreased compared with that in the control group, the ballooning degeneration of hepatocytes was more intensive and appeared extensively. Mallory bodies and the condensation of subcellular organelles were also sporadically observed (Figure 5(c) and its inset) as were small necrotic foci. No fibrosis was evident. In several cases, TNF- $\alpha$ expression was aberrantly observed in the hepatocytes of the perivenular and periportal areas; however, it was not seen in Kupffer cells (Figure 5(d)). Evaluation of liver pathology is shown in Table 2.

\section{DISCUSSION}

Metabolic syndrome is a combination of disorders that increase the risk of developing cardiovascular disease and diabetes $[1,2]$. It affects one in five people, and its prevalence increases with age. Some 


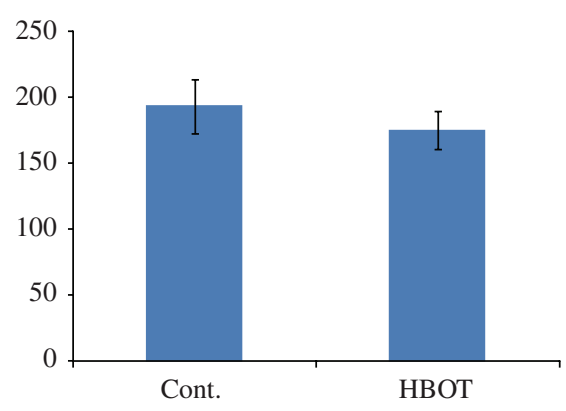

(a) T-chol

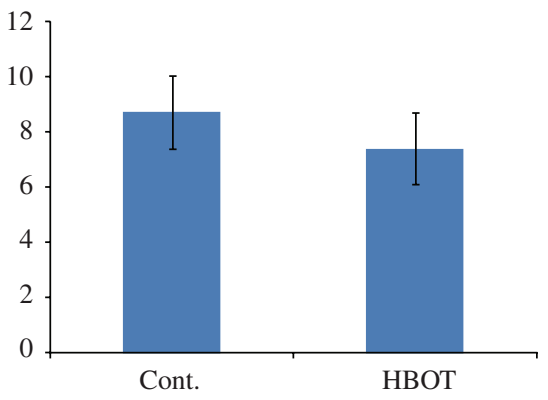

(c) VLDL-chol

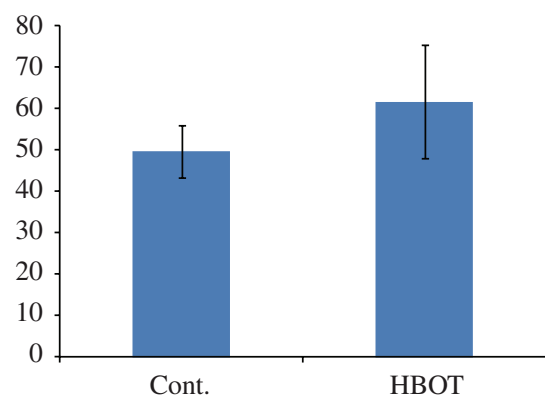

(b) TG

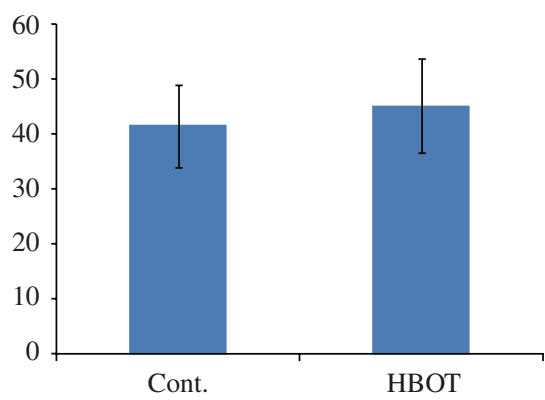

(d) LDL-chol

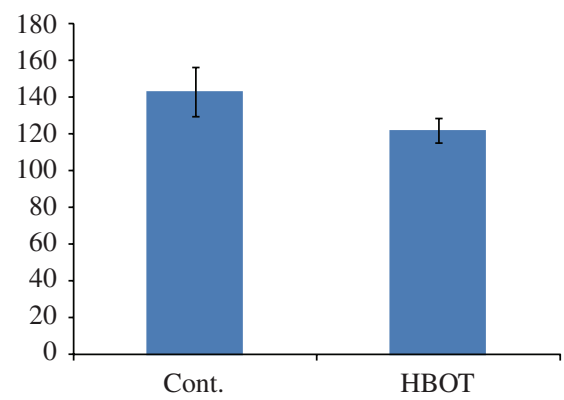

(e) HDL-chol

FIGURE 3: The serum lipid levels of each group (Student's $t$-test).

studies have estimated its prevalence in the USA to be up to $25 \%$ of the population. Metabolic syndrome is also known as metabolic syndrome $\mathrm{X}$, insulin resistance syndrome, and visceral fat syndrome. Usually it involves the development of visceral fat, after which the adipocytes (fat cells) of the visceral fat increase the plasma levels of TNF- $\alpha$ and alter the levels of a number of other substances (e.g., adiponectin, resistin, PAI-1). TNF- $\alpha$ has been shown not only to cause the production of inflammatory cytokines but also to trigger cell signaling by interacting with the TNF- $\alpha$ receptor, which may lead to insulin resistance. The progression from visceral fat to increased TNF- $\alpha$ to insulin resistance has some parallels to the human development of metabolic syndrome $[26,27]$. NASH is thought to be the phenotype of metabolic syndrome in the liver, and the number of patients with the condition is constantly increasing. In Japan, 10-30\% of population is considered to have NAFLD accompanied by metabolic syndrome, and $10 \%$ is presumed to have NASH, a progressive pathologic lesion [5]. Although there are many unclear points about the pathogenetic mechanism of NASH, a two-hit theory in which fat is first accumulated in hepatocytes by overeating, hyperlipidemia, and so forth, then the pathologic condition is induced to progress by a second hit, such as oxidative stress, is widely accepted $[4,6]$. No definite remedy for metabolic syndrome or NASH has been established, and dietary counseling and therapeutic exercise are considered as the first options 


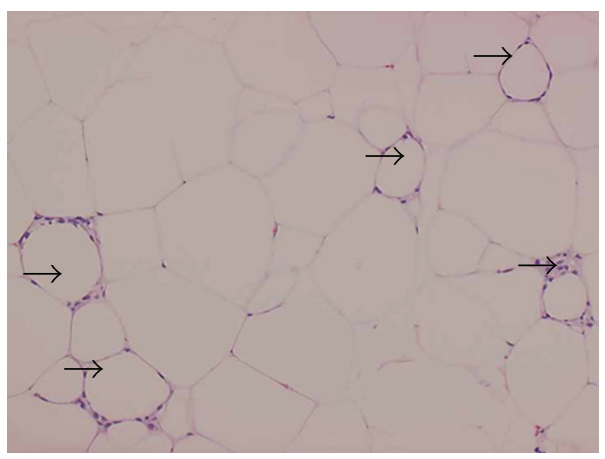

(a)

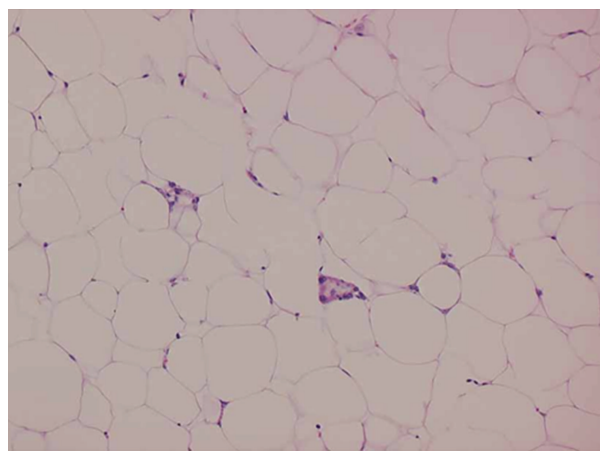

(c)

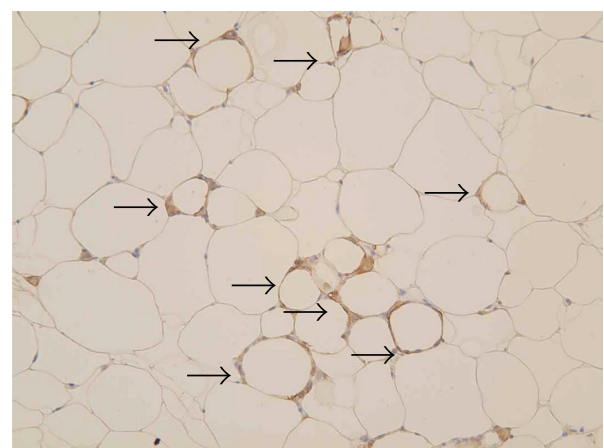

(b)

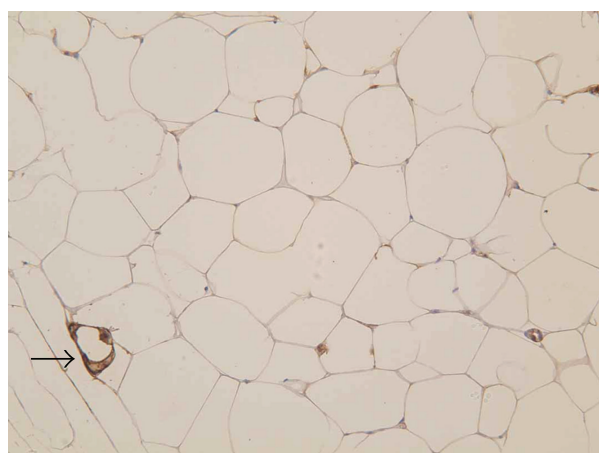

(d)

FIGURE 4: (a), (b) Visceral fat of control group. Adipocytes are markedly enlarged. Crown-like structures are frequently observed (arrows) $(\times 200)((\mathrm{a}) \mathrm{H}$ and E staining, (b) Mac-2 immunostaining as a macrophage marker) (c), (d) Visceral fat of HBOT group. Adipocytes are relatively small. Crown-like structures are rarely observed (arrows). (×200) ((c) H and E staining, (d) Mac-2 immunostaining as a macrophage marker).

$[28,29]$. However, a continuous strict adaptation is difficult with remedies based on lifestyle improvement, and the response rate is not always high. Large-scale clinical trials of pioglitazone for patients with NASH caused by metabolic syndrome have been performed; however, the reported effects are contradictory. For example, simultaneous weight gains together with improvements in damage have been reported [30-32].

Hyperbaric oxygen therapy is a remedy that improves hypoxia by inhaling hyperbaric oxygen in an environment in which the atmospheric pressure is higher than that of the normal atmosphere. Although it is widespread as a remedy for carbon monoxide poisoning and sudden deafness, its application for other purposes, such as in the fields of orthopedics and sports medicine, is also being widely investigated [7-11]. We have reported that the oxidative stress raising effects of HBOT may affect the "pathogenic cells" involved in autoimmune disease or hematopoietic malignancies selectively, using animal models and cultured cells $[12,13]$. This indicates the potential of HBOT being applied to various diseases through a mechanism other than medication. In this study, we proposed the hypothesis that HBOT improves various symptoms of metabolic syndrome and attempted in vivo verification in an animal model. The animal model used was the MSG mouse, which shows various symptoms of metabolic syndrome with near-natural development and is known to show the symptoms of central obesity, hyperlipidemia, type- 2 diabetes, and NASH in succession after 12 weeks of age [23, 24]. In the current study, our research was limited to obesity, hyperlipidemia, and NASH, and the benefits of HBOT on metabolic syndrome were examined.

As a result, although a weight gain of about $3 \mathrm{~g}$ in the first phase of the HBOT treatment period (two weeks) was seen in the control group, weight loss was observed with eight out of ten mice in the HBOTtreated group. The mean weight of the ten animals was $56.8 \mathrm{~g}$ before HBOT treatment, and it decreased to $53.6 \mathrm{~g}$ after HBOT treatment that is, a mean of $3.2 \mathrm{~g}$ weight loss. When HBOT treatment was stopped, the 


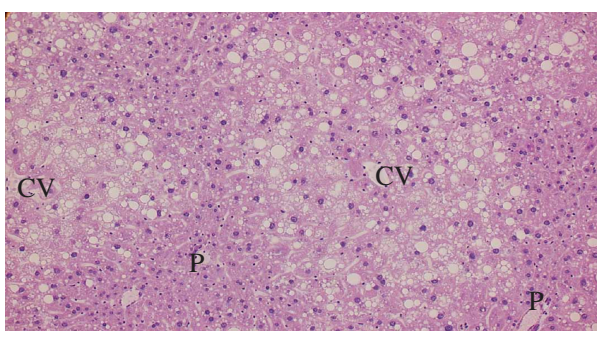

(a)

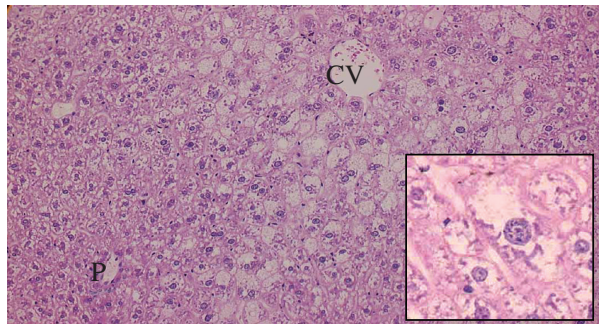

(c)

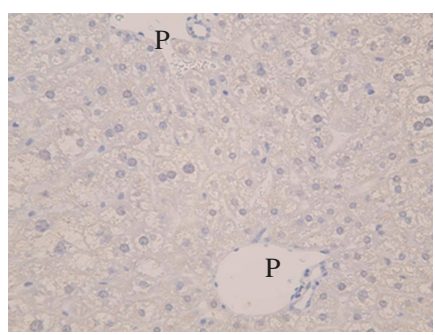

(b)

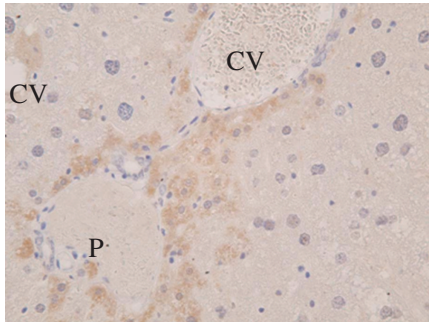

(d)

FIGURE 5: (a), (b) Liver of the control group. (a) $\mathrm{H}$ and $\mathrm{E}$ staining, $\times 200$ magnification. In Zone3-Zone2, moderate fatty changes were observed. Ballooning degeneration was sporadically observed. (b) TNF- $\alpha$ immunostaining, $\times 400$ magnification. No TNF- $\alpha$ expression was seen in hepatocytes or Kupffer cells. (P: portal tracts, CV: central vein) (c), (d) Liver of the HBOT group. (c) $\mathrm{H}$ and $\mathrm{E}$ staining, $\times 200$ magnification. Fatty changes were less severe, while marked ballooning degeneration was observed. Mallory bodies were seen sporadically (inset). (d) TNF- $\alpha$ immunostaining, $\times 400$ magnification. TNF- $\alpha$ expression was observed in the hepatocytes of the perivenular and periportal areas; however, it was not seen in Kupffer cells. (P: portal tracts, CV: central vein).

rate of weight increase of the mice recovered to the same extent as seen in the untreated mice; however, the mean body weight among ten mice decreased from $57.9 \mathrm{~g}$ to $51.7 \mathrm{~g}$ (by $6.2 \mathrm{~g}$ ) during the second phase of HBOT treatment. Although we observed the body weight reduction in the 1st phase of HBOT, the weight of body developed slightly after two weeks of HBOT. Therefore, we performed another course of HBOT just as what we will try in the clinics for patients with different kinds of sickness. We expected further weight reduction to our mice, and the results were positive. However, the burst of oxidative stress might induce normal hepatocytes but not the fat cells undergoing apoptosis. This may be consistent with our previous experiments that cells with different origins have different threshold to oxidative stress-induced apoptosis [13]. Furthermore, the interesting point is that cells with higher proliferative rate such as cancer cells have a lower threshold to oxidative stress induced by hyperbaric oxygen treatment, and $\mathrm{HBO}_{2}$-induced apoptosis of cancer cells was through the intracellular accumulation of $\mathrm{H}_{2} \mathrm{O}_{2}$ and $\mathrm{O}_{2}{ }^{-}{ }^{-}$as well as the involvement of phosphorylation of p38 MAPK [13]. Our unpublished data further demonstrated that $\mathrm{HBO}_{2}$ treatment effectively suppressed tumor growth from lung cancer transferred SCID mice after 14 days $\left.{ }^{* *} P<0.01\right)$ and 28 days $\left({ }^{* *} P<0.01\right)$ of tumor transfer compared with control mice. The survival rate of $\mathrm{HBO}_{2}$ treatment mice was increased significantly compared with control mice. Since oxidative stress is closely related to the development and progression of metabolic syndrome and cardiovascular disease; therefore, we should be more careful to improve this side effect: firstly, we could reduce the pressure and duration in the 2nd phase of HBOT; secondly, supplement of optimal antioxidants could be prescribed in the 2nd phase of HBOT. Future studies should be done in order to avoid the HBOT-mediated deterioration of liver histology.

Although the mechanism of obesity in MSG mice is not yet clear, weight gain due to a fall in energy consumption is suggested. In our study, it also turned out that, when MSG mice were bred with $75 \%$ 
TABLE 2: NAS score and fibrosis.

\begin{tabular}{lccccc}
\hline & Steatosis & Lobular inflammation & Hepatocyte ballooning & NAS & Fibrosis \\
\hline HBOT-1 & 1 & 2 & 2 & 5 & 0 \\
HBOT-2 & 1 & 1 & 1 & 3 & 0 \\
HBOT-3 & 1 & 1 & 2 & 4 & 0 \\
HBOT-4 & 0 & 1 & 2 & 3 & 0 \\
HBOT-5 & 1 & 1 & 2 & 4 & 0 \\
HBOT-6 & 1 & 1 & 1 & 3 & 0 \\
HBOT-7 & 1 & 1 & 2 & 4 & 0 \\
HBOT-8 & 2 & 1 & 2 & 5 & 0 \\
HBOT-9 & 1 & 1 & 1 & 3 & 0 \\
HBOT-10 & 2 & 1 & 1 & 4 & 0 \\
\hline Cont-1 & 1 & 1 & 1 & 4 & 0 \\
Cont-2 & 2 & 1 & 1 & 2 & 0 \\
Cont-3 & 1 & 1 & 0 & 4 & 0 \\
Cont-4 & 2 & 1 & 1 & 4 & 0 \\
Cont-5 & 1 & 1 & 2 & 4 & $1 \mathrm{~A}$ \\
Cont-6 & 2 & 1 & 1 & 3 & 0 \\
Cont-7 & 1 & 1 & 1 & 4 & 0 \\
Cont-8 & 2 & 1 & 0 & 3 & 0 \\
Cont-9 & 2 & 1 & & & 4 \\
\hline
\end{tabular}

of their usual food intake, the increase in weight and liver damage were identical to those seen in the mice with normal food intakes (unpublished data). Although a future detailed examination is required for the mechanism of weight loss induced by HBOT, one possibility is that rapid improvement in systemic metabolism due to the sufficient supply of oxygen to peripheral tissues induced the weight loss. Another possibility is that the prevention of overactivity of pathogenic cells in visceral fat and the induction of apoptosis cause the weight loss.

In test concerning the levels of lipid-associated molecules in serum, slight increases in triglyceride and LDL-chol were observed in the HBOT-treated group. Since the visceral fat and fat cumulative dosage in the liver decreased in the HBOT-treated group, the lipids may have migrated into the blood from the cells storing them. On the other hand, although the lipid cumulative dosage in hepatocyte was decreased in the liver, the grade of the hepatocellular damage became getting worse. While HBOT supplies sufficient oxygen to local sites, it is known to induce a strong oxidative stress. Oxidative stress is considered to be a key accelerator of cell damage and oncogenesis as the second hit of NASH [4-6]. It was supposed that oxidative stress also played a role in the hepatocellular damage observed in the present study. This indicates that HBOT is a "double-edged blade" as it has the advantage of improving obesity and the fault of causing organ damage by increasing oxidative stress.

Patients with metabolic syndrome usually have multiple diseases, and there are often many restrictions on the medication they can take. Although HBOT has the potential of becoming an effective remedy in support of diet restriction and therapeutic exercises, for practical use, further techniques, such as suppressing the harmful effects of oxidative stress, are indispensable. It is believed that the intake of antioxidants, such as polyphenol and vitamin E, is effective in reducing oxidative stress [33, 34]. It may be possible to extract only the benefits of HBOT by taking antioxidants as supplements, before and after 
HBOT treatment. It is important to examine different conditions of HBOT treatment in order to determine the most effective conditions for treating metabolic syndrome.

\section{AUTHOR CONTRIBUTION}

All authors participated in the design, interpretation of the studies, analysis of the data, and review of the paper. K. Tsuneyama, Y. C. Chen, M. Kujimoto, and S. Y. Chen conducted the experiments, Y. Sasaki, W. Suzki, T. Shimada, S. Iizuka, M. Nagata, and M. Aburada supplied critical reagents and experimental animals, and K. Tsuneyama, Y. C. Chan, M. Fujimoto, and S. Y. Chen wrote the paper. Koichi Tsuneyama and Yen-Chen Chen contributed equally to this work.

\section{ACKNOWLEDGMENTS}

The authors thank Tokimasa Kumada and Takeshi Nishida for their technical assistance. This study was supported by a research grant from the Interchange association of JAPAN (2008).

\section{REFERENCES}

[1] L. Guize, B. Pannier, F. Thomas, K. Bean, B. Jégo, and A. Benetos, "Recent advances in metabolic syndrome and cardiovascular disease," Archives of Cardiovascular Diseases, vol. 101, no. 9, pp. 577-583, 2008.

[2] M. A. Cornier, D. Dabelea, T. L. Hernandez et al., "The metabolic syndrome," Endocrine Reviews, vol. 29, no. 7, pp. 777-822, 2008.

[3] P. D. Berk, "Regulatable fatty acid transport mechanisms are central to the pathophysiology of obesity, fatty liver, and metabolic syndrome," Hepatology, vol. 48, no. 5, pp. 1362-1376, 2008.

[4] G. C. Farrell and C. Z. Larter, "Nonalcoholic fatty liver disease: from steatosis to cirrhosis," Hepatology, vol. 43, no. 2, pp. S99-S112, 2006.

[5] J. M. Clark, "The epidemiology of nonalcoholic fatty liver disease in adults," Journal of Clinical Gastroenterology, vol. 40, supplement 1, pp. S5-S10, 2006.

[6] M. M. Yeh and E. M. Brunt, "Pathology of nonalcoholic fatty liver disease," American Journal of Clinical Pathology, vol. 128, no. 5, pp. 837-847, 2007.

[7] M. D'Agostino Dias, B. Fontes, R. S. Poggetti, and D. Birolini, "Hyperbaric oxygen therapy: types of injury and number of sessions-a review of 1506 cases," Undersea \& Hyperbaric Medicine, vol. 35, no. 1, pp. 53-60, 2008.

[8] K. C. Huang, W. H. Hsu, K. T. Peng, T. J. Huang, and R. W. W. Hsu, "Hyperbaric oxygen therapy in orthopedic conditions: an evaluation of safety," Journal of Trauma, vol. 61, no. 4, pp. 913-917, 2006.

[9] M. Kawashima, H. Tamura, I. Nagayoshi, K. Takao, K. Yoshida, and T. Yamaguchi, "Hyperbaric oxygen therapy in orthopedic conditions," Undersea and Hyperbaric Medicine, vol. 31, no. 1, pp. 155-162, 2004.

[10] G. Germain, J. Delaney, G. Moore, P. Lee, V. Lacroix, and D. Montgomery, "Effect of hyperbaric oxygen therapy on exercise-induced muscle soreness," Undersea and Hyperbaric Medicine, vol. 30, no. 2, pp. 135-145, 2003.

[11] S. Babul and E. C. Rhodes, "The role of hyperbaric oxygen therapy in sports medicine," Sports Medicine, vol. 30, no. 6, pp. 395-403, 2000.

[12] S. Y. Chen, Y. C. Chen, J. K. Wang et al., "Early hyperbaric oxygen therapy attenuates disease severity in lupusprone autoimmune (NZB × NZW) F1 mice," Clinical Immunology, vol. 108, no. 2, pp. 103-110, 2003.

[13] Y. C. Chen, S. Y. Chen, P. S. Ho et al., "Apoptosis of T-leukemia and B-myeloma cancer cells induced by hyperbaric oxygen increased phosphorylation of p38 MAPK," Leukemia Research, vol. 31, no. 6, pp. 805-815, 2007.

[14] Y. Kamada, T. Takehara, and N. Hayashi, “Adipocytokines and liver disease," Journal of Gastroenterology, vol. 43, no. 11, pp. 811-822, 2008. 
[15] I. Murano, G. Barbatelli, V. Parisani et al., "Dead adipocytes, detected as crown-like structures, are prevalent in visceral fat depots of genetically obese mice," Journal of Lipid Research, vol. 49, no. 7, pp. 1562-1568, 2008.

[16] K. J. Strissel, Z. Stancheva, H. Miyoshi et al., "Adipocyte death, adipose tissue remodeling, and obesity complications," Diabetes, vol. 56, no. 12, pp. 2910-2918, 2007.

[17] S. Unfirer, A. Kibel, and I. Drenjancevic-Peric, "The effect of hyperbaric oxygen therapy on blood vessel function in diabetes mellitus," Medical Hypotheses, vol. 71, no. 5, pp. 776-780, 2008.

[18] N. S. Al-Waili, G. J. Butler, J. Beale et al., "Influences of hyperbaric oxygen on blood pressure, heart rate and blood glucose levels in patients with diabetes mellitus and hypertension," Archives of Medical Research, vol. 37, no. 8, pp. 991-997, 2006.

[19] K. Yasuda, N. Aoki, T. Adachi et al., "Hyperbaric exposure with high oxygen concentration inhibits growthassociated increase in the glucose level of diabetic Goto-Kakizaki rats," Diabetes, Obesity and Metabolism, vol. 8, no. 6, pp. 714-715, 2006.

[20] K. Nomoto, K. Tsuneyama, H. Takahashi, Y. Murai, and Y. Takano, "Cytoplasmic fine granular expression of 8hydroxydeoxyguanosine reflects early mitochondrial oxidative DNA damage in nonalcoholic fatty liver disease," Applied Immunohistochemistry and Molecular Morphology, vol. 16, no. 1, pp. 71-75, 2008.

[21] Y. Kadokawa, K. Ohba, K. Omagari et al., "Intracellular balance of oxidative stress and cytoprotective molecules in damaged interlobular bile ducts in autoimmune hepatitis and primary biliary cirrhosis: in situ detection of 8-hydroxydeoxyguanosine and glutathione-S-transferase-pi," Hepatology Research, vol. 37, no. 8, pp. 620-627, 2007.

[22] V. M. Victor and M. De La Fuente, "Several functions of immune cells in mice changed by oxidative stress caused by endotoxin," Physiological Research, vol. 52, no. 6, pp. 789-796, 2003.

[23] M. Nagata, W. Suzuki, S. Iizuka et al., "Type 2 diabetes mellitus in obese mouse model induced by monosodium glutamate," Experimental Animals, vol. 55, no. 2, pp. 109-115, 2006.

[24] Y. Nakanishi, K. Tsuneyama, M. Fujimoto et al., "Monosodium glutamate (MSG): a villain and promoter of liver inflammation and dysplasia," Journal of Autoimmunity, vol. 30, no. 1-2, pp. 42-50, 2008.

[25] Y. Sasaki, W. Suzuki, T. Shimada et al., "Dose dependent development of diabetes mellitus and non-alcoholic steatohepatitis in monosodium glutamate-induced obese mice," Life Sciences, vol. 85, no. 13-14, pp. 490-498, 2009.

[26] D. E. Kleiner, E. M. Brunt, M. Van Natta et al., "Design and validation of a histological scoring system for nonalcoholic fatty liver disease," Hepatology, vol. 41, no. 6, pp. 1313-1321, 2005.

[27] I. Copaci, L. Micu, and M. Voiculescu, "The role of cytokines in non-alcoholic steatohepatitis. A systematic review," Journal of Gastrointestinal and Liver Diseases, vol. 15, no. 4, pp. 363-373, 2006.

[28] N. Rafiq and Z. M. Younossi, "Effects of weight loss on nonalcoholic fatty liver disease," Seminars in Liver Disease, vol. 28, no. 4, pp. 427-433, 2008.

[29] M. Cave, I. Deaciuc, C. Mendez et al., "Nonalcoholic fatty liver disease: predisposing factors and the role of nutrition," Journal of Nutritional Biochemistry, vol. 18, no. 3, pp. 184-195, 2007.

[30] G. P. Aithal, J. A. Thomas, P. V. Kaye et al., "Randomized, placebo-controlled trial of pioglitazone in nondiabetic subjects with nonalcoholic steatohepatitis," Gastroenterology, vol. 135, no. 4, pp. 1176-1184, 2008.

[31] M. Fujimoto, K. Tsuneyama, M. Kainuma et al., "Evidence-based efficacy of kampo formulas in a model of non alcoholic fatty liver," Experimental Biology and Medicine, vol. 233, no. 3, pp. 328-337, 2008.

[32] B. Balas, R. Belfort, S. A. Harrison et al., "Pioglitazone treatment increases whole body fat but not total body water in patients with non-alcoholic steatohepatitis," Journal of Hepatology, vol. 47, no. 4, pp. 565-570, 2007.

[33] E. Reboul, S. Thap, F. Tourniaire et al., "Differential effect of dietary antioxidant classes (carotenoids, polyphenols, vitamins C and E) on lutein absorption," British Journal of Nutrition, vol. 97, no. 3, pp. 440-446, 2007.

[34] K. J. Anderson, S. S. Teuber, A. Gobeille, P. Cremin, A. L. Waterhouse, and F. M. Steinberg, "Walnut polyphenolics inhibit in vitro human plasma and LDL oxidation," Journal of Nutrition, vol. 131, no. 11, pp. 2837-2842, 2001. 


\section{This article should be cited as follows:}

Koichi Tsuneyama, Yen-Chen Chen, Makoto Fujimoto, Yoshiyuki Sasaki, Wataru Suzuki, Tsutomu Shimada, Seiichi Iizuka, Mitsunobu Nagata, Masaki Aburada, and Shao-Yuan Chen, "Advantages and Disadvantages of Hyperbaric Oxygen Treatment in Mice with Obesity Hyperlipidemia and Steatohepatitis," TheScientificWorldJOURNAL, vol. 11, pp. 2124-2135, 2011. 


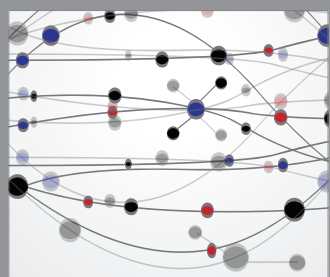

The Scientific World Journal
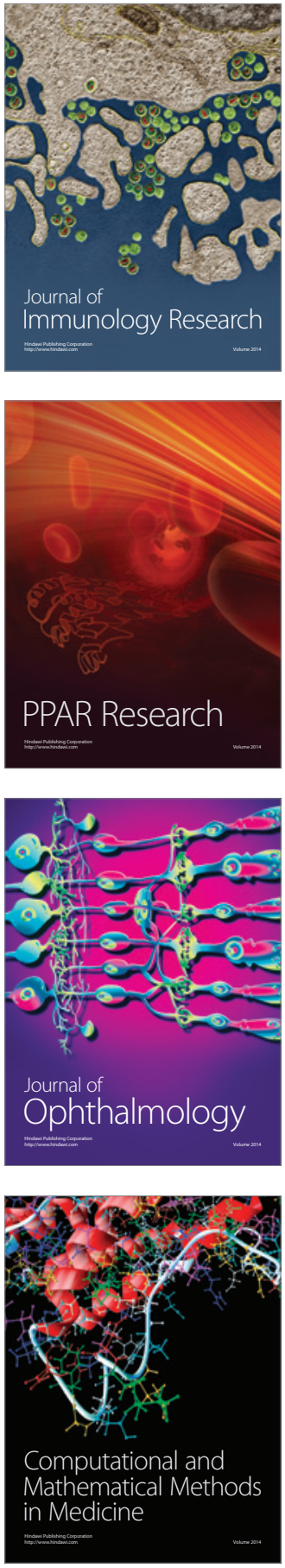

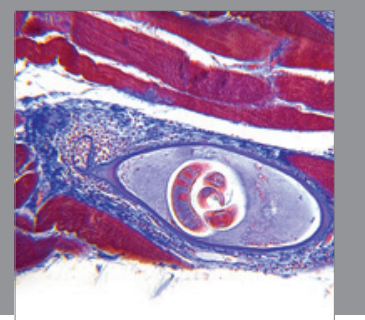

Gastroenterology

Research and Practice
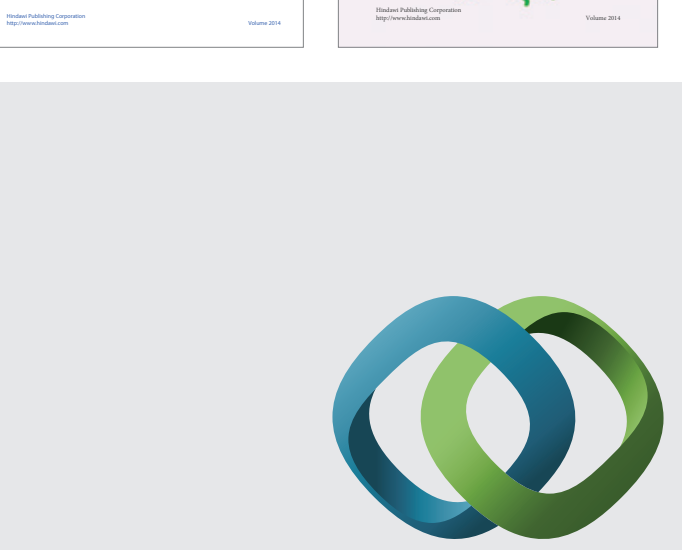

\section{Hindawi}

Submit your manuscripts at

http://www.hindawi.com
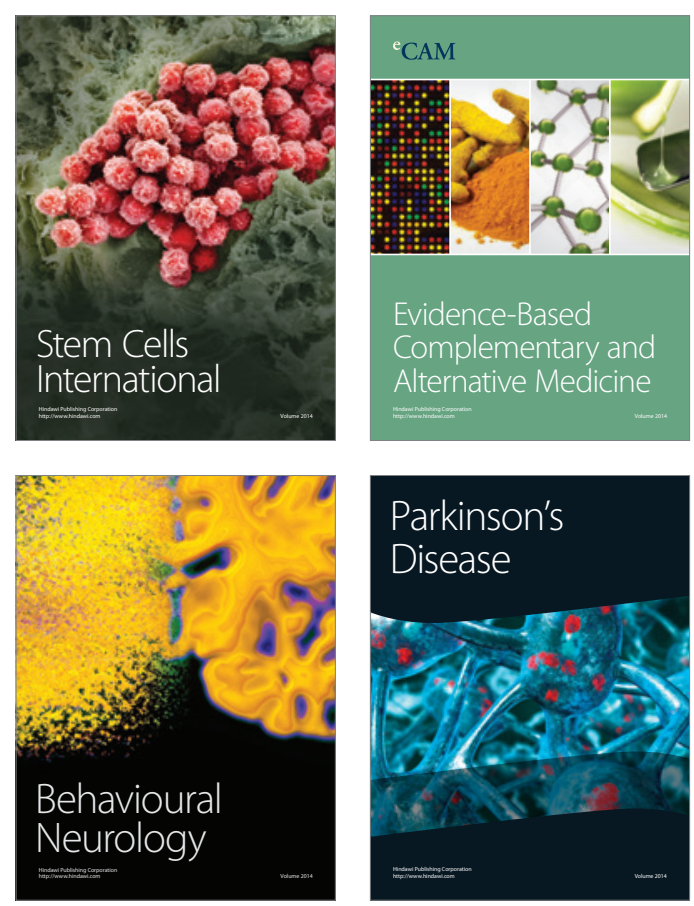

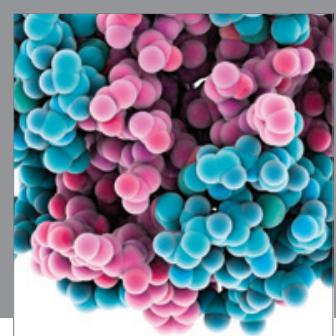

Journal of
Diabetes Research

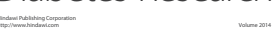

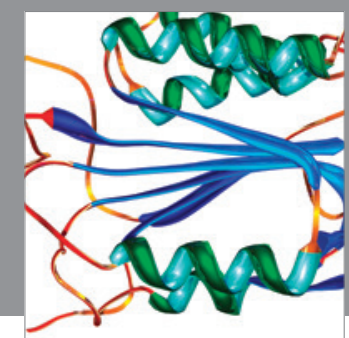

Disease Markers
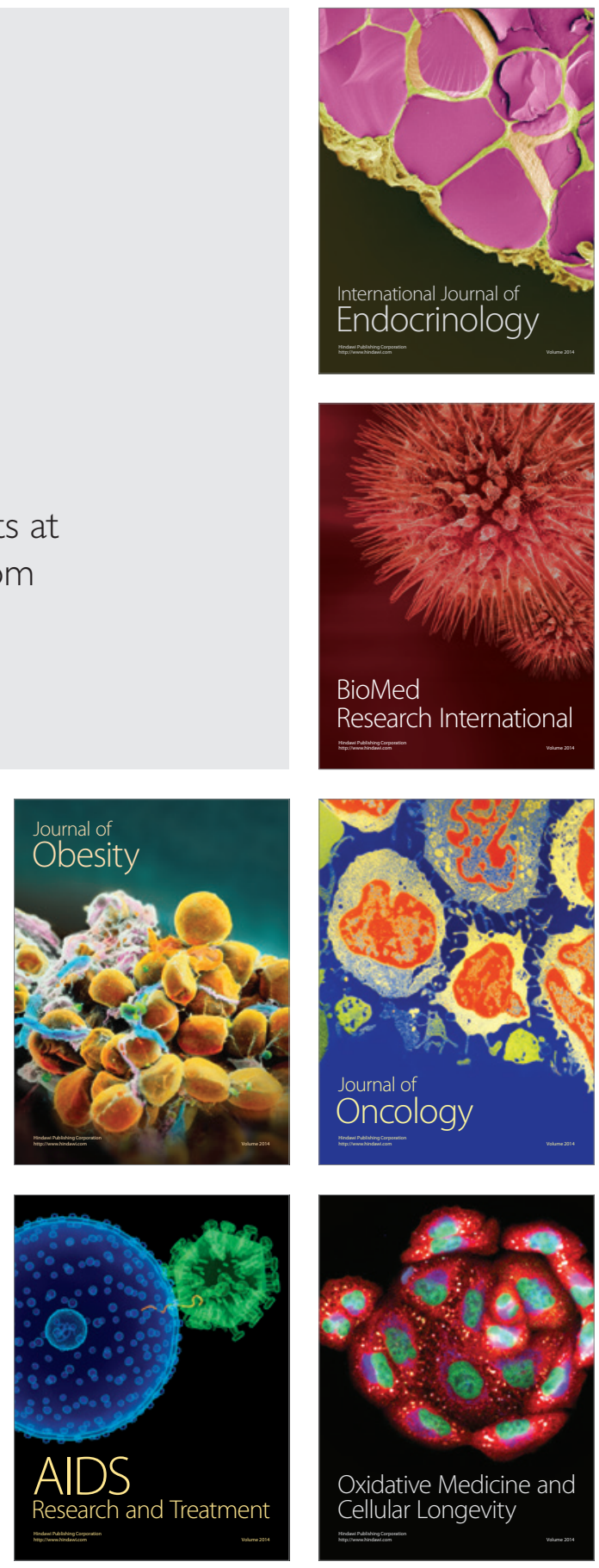